\title{
Ebenezer: um modelo para prevenção ubíqua de acidentes de trabalho
}

\author{
Isaías Machado da Rosa ${ }^{1}$ and Jorge Luis Victória Barbosa ${ }^{2}$ \\ ${ }^{12}$ Universidade do Vale do Rio dos Sinos (Unisinos) \\ *isaiasmachadodarosa@gmail.com; jbarbosa@unisinos.br
}

Submetido: 20/12/2017. Revisado: 23/03/2018. Aceito: 14/04/2018.

\begin{abstract}
Resumo
Neste artigo é proposto Ebenezer, um modelo de computação ubíqua para prevenir acidentes em um ambiente de trabalho inteligente. Sua principal característica é a notificação aos trabalhadores e aos usuários em geral sobre a presença de perigos nos arredores, para evitar riscos de acidentes. A aplicação e validação foram realizadas com os trabalhadores em seus ambientes de trabalho e por especialistas na área de gestão industrial e segurança do trabalho. Uma pesquisa foi aplicada baseada no modelo de aceitação de tecnologia, resultando em $97 \%$ de aceitação na facilidade de uso e $100 \%$ na utilidade percebida. Os resultados promissores demonstram que a solução é útil, permitindo que esta tecnologia seja aprimorada, para se tornar um acessório de segurança para evitar acidentes com usuários e trabalhadores.
\end{abstract}

Palavras-Chave: Atividade ocupacional; computação ubíqua; prevenção de acidentes; riscos.

\begin{abstract}
This paper proposes Ebenezer, a ubiquitous computing model to prevent accidents in an intelligent work environment, with the main characteristic being the notification to workers and even to users in general about the presence of hazards in their surroundings, in order to prevent the risk of accidents. The application and validation were carried out with the workers in their work places and by specialists in the area of industrial management and safety at work. In which a research was applied based on the Technology Acceptance Model, resulting in $97 \%$ acceptance in the ease of use and $100 \%$ in the perceived utility. The promising results demonstrate that the solution is useful, allowing this technology to be improved to become a safety accessory to avoid accidents with users and workers.
\end{abstract}

Key words: Accidents prevention; occupational activity; scratchs; ubiquitous computing.

\section{Introdução}

Segundo o sistema de Informação de Agravos de Notificação (Bastos-Ramos et al.; 2015), os acidentes de trabalho aumentaram cerca de $420 \%$ de 2011 em relação ao período de 2007, também a Pesquisa Nacional de Saúde (Maia et al.; 2016) realizada em 2013, divulgou que aproximadamente $3 \%$ dos trabalhadores sofreram acidentes. Os programas de prevenção de acidentes convencionais, adotados nas empresas, órgãos ou instituições, promovem ações na tentativa de reduzir a ocorrência de acidentes (Simonelli and Camarotto; 2011), porém estas medidas tornam-se fragilizadas, devido ao fator comportamental dos empregados (Simonelli and Camarotto; 2011), que é a principal causa dos acidentes de trabalho (Almeida and Jackson Filho; 2007). Diante da lacuna aberta nestes programas de prevenção, estão sendo testados para este propósito, aplicações utilizando a computação ubíqua (Kritzler et al.; 2015), conhecidas na literatura como ambientes de trabalho inteligentes.

Segundo os autores Podgórski et al. (2016), os 
ambientes de trabalho inteligentes, do original smart working environments (SWE), são locais sob determinadas condições, nos quais os trabalhadores realizam as suas atividades, onde são monitorados os parâmetros fisiológicos, ambientais e objetos (máquinas, ferramentas, dispositivos de segurança, equipamentos de proteção individual), por meio das tecnologias aplicadas em ambientes inteligentes (Internet das coisas, sistemas cibernéticos). Os SWE objetivam garantir a segurança e o conforto dos trabalhadores, através da análise de contexto, aplicando medidas normativas, de prevenção, proteção e controle sobre os níveis de risco, na esfera de trabalho e dos trabalhadores, individual ou coletivamente.

O modelo proposto foi analisado através do estudo de trabalhos relacionados às abordagens que utilizam a computação ubíqua na prevenção de acidentes com trabalhadores, considerando as estratégias utilizadas de monitoramento do ambiente, da atividade ocupacional, dos riscos e os métodos de controle para a prevenção dos acidentes. $O$ trabalho em questão é baseado na abordagem de uma pesquisa qualitativa, na qual designa-se a compressão de um grupo social (Gerhardt and Silveira; 2009), no caso específico os trabalhadores realizando as suas atividades.

O trabalho está estruturado da seguinte forma: a seção 2 trata sobre os trabalhos relacionados, considerando as suas abordagens para fins comparativos. Na seção 3 é descrito o modelo; a seção 4 descreve a implementação e avaliação do modelo em dois cenários. Na seção 5 são apresentados os resultados obtidos na pesquisa e por fim a conclusão a respeito do trabalho, contribuições e trabalhos futuros.

\section{Trabalhos relacionados}

Esta seção descreve os trabalhos relacionados baseados em soluções que utilizam a computação ubíqua ou alguns de seus conceitos, para a prevenção de acidentes nos ambientes de trabalho. Estes trabalhos foram selecionados através da consulta na base de dados de periódicos da CAPES, Google Acadêmico, Science Direct, IEEE Xplore, ACM Digital Library. Para esta consulta, utilizando as palavras chaves "ubiquitous accident prevention", "ubiquitous accident prevention work", "Ubiquitous computing work place", "Smart factory systems", "smart working environment" e aplicado os filtros por relevância e por datas, resultaram os seguintes trabalhos.

Collision accident (Chae and Yoshida; 2010) é uma aplicação que utiliza tags RFID, para identificar as entidades envolvidas (equipamentos, pessoa ou localização), no canteiro de obras a fim de prevenir colisões dos equipamentos pesados (escavadeiras e guindastes) com os trabalhadores. Baseado em uma lógica de associações de causas de acidentes passados, na qual o servidor administrativo realiza a estimativa do nível de risco de colisão, levando em consideração o raio de ação dos equipamentos em relação à proximidade com outras pessoas, e conforme o nível de risco envia sinais de alerta para as partes interessadas, que estão em uma situação de perigo.

Proactive prevention (Yang et al.; 2012) implementa um sistema de identificação de precursores de acidentes como veículos, trabalhadores e materiais para uma segurança pró-ativa, restringindo o acesso aos materiais ou veículos, somente a trabalhadores autorizados no canteiro de obras da construção civil. Para a identificação dos precursores de acidentes, foi utilizado sensores Radio-Frequency IDentification (RFID) integrados a estrutura de rede ZigBee.

Risk management (Fugini et al.; 2012) é um protótipo de simulação que mostra o nível de risco que estão expostos os trabalhadores. O nível de risco é visualizado no formato de mapa de cores, onde as cores mais claras indicam os locais seguros no mapa do ambiente de trabalho, auxiliando assim, os trabalhadores a enfrentarem o perigo e indicando soluções imediatas.

Passage construction (Ding et al.; 2013) é um sistema de alertas de segurança para a prevenção de acidentes em construção subterrânea. Baseado em modelos matemáticos e mecânicos, mineração e fusão dos dados e conhecimento de especialistas, são aplicadas inferências sobre os dados monitorados via internet das coisas, relativo as entidades participantes (trabalhadores, temperatura, pressão e deslocamento de estruturas e ambientes), para verificar se as condições ou comportamentos das entidades atingiram um nível inaceitável de risco.

Gates portals (Kelm et al.; 2013) é uma aplicação não intrusiva, que verifica a conformidade dos PPEs (Personal Protective Equipment) dos trabalhadores ao acessarem os canteiros de obras pelas catracas. $\mathrm{O}$ sistema faz a verificação através do sensoriamento RFID fixado aos PPEs e exibe a mensagem de conformidade ou não dos equipamentos utilizados.

Workplace safety (Kritzler et al.; 2015) é um aplicativo para assegurar que o trabalhador execute as tarefas usando os equipamentos de proteção individuais (EPIs) adequados nas estações de trabalho, através de alertas visualizados no Smartwatch utilizado pelo trabalhador, onde consta a presença ou não destes EPIs. Para o monitoramento das estações de trabalho e dos EPIs é utilizado o beacon, que é uma tecnologia para sistemas de localização em ambientes fechados. O beacon envia pacotes continuos via bluetooth, contendo o identificador único universal (UUID) e a intensidade do sinal recebido (RSSI) (Lin et al.; 2015).

Adaptive security (Fugini et al.; 2016) é um sistema para gerenciar os riscos de acidentes, mostrando em um painel de controle (dashbord) o mapa do ambiente de trabalho, permitindo aos gestores, de acordo com as políticas de acesso baseadas em papéis, a visualização dos trabalhadores quando eles estiverem expostos aos riscos de acidentes e o acesso aos recursos, como a liberação para o acionamento de um ventilador para eliminar o acumulo de gás detectado em um ambiente.

Early warning (Yi et al.; 2016) é um aplicativo de alertas preventivos para trabalhos em ambiente quentes e úmidos na construção civil. O modelo compara o batimento cardíaco do trabalhador, com o índice de percepção de esforço, obtido através de redes neurais, considerando a temperatura e umidade do ambiente e o perfil fisiológico do 
trabalhador. Neste trabalho foram utilizadas as tecnologias para transmissão de dados Global System for Mobile communication (GSM) e Bluetooth, para monitoramento e alertas, a pulseira inteligente e o smartphone.

RAMIRES (Teimourikia and Fugini; 2016) implementa o mapa de riscos em um painel de controle (dashboard), para auxiliar na gestão de segurança em ambientes de trabalho inteligentes, através de uma ontologia baseada nas normas da agência OSHA (Occupational Safety and Health Administration) e do modelo utilizado em sistemas autônomos MAPE-K (Monitor-Analyze-PlanExecute and Knowledge)(Computing et al.; 2006).

Este sistema monitora as entidades envolvidas no SWE através do sensoriamento pela Internet das coisas. Ele avalia os riscos para compreensão das consequências e das probabilidades de ocorrência, para tomada de decisão sobre a prioridade e a intensidade destes riscos; planeja as estratégias de prevenção dos riscos de acidentes; executa simultaneamente as estratégias automáticas; dá suporte à operação manual e controla o acesso dos atores à informação representada em sua ontologia.

Para fins comparativos, foram adotados parâmetros que envolvem um ambiente de trabalho inteligente (Podgórski et al.; 2016):

- Abrangência: Qual a área de abrangência suportada para notificar os usuários sobre os perigos: a) "indústria"; b) "setor específico" como portarias, estações de trabalho; c) construção "civil"; d) construção "subterrânea"; e) "diversos", no caso de abranger diversos ambientes de trabalho.

- Dispositivo: Qual o tipo de dispositivo utilizado no trabalho: a) "próprio" no caso de um hardware dedicado, desenvolvido para o trabalho; b) "smartphone"; c) "smartwatch"; d) web "dashboard"; e) "servidor".

- Notificação: Qual o tipo de artefato utilizado no trabalho para alertar sobre os perigos: a) "luminoso"; b) "textual"; c) "imagem"; d) "sonoro"; e) "tela", mostra o mapa de risco e as entidades envolvidas.

- Prevenção: Qual o método de prevenção adotado: a) "individual"; b) "coletivo"; c) "ambos".

- Suporte: Qual o tipo de comunicação realizada entre o usuário e o sistema, caso ele forneça suporte ao usuário em uma situação que necessite de ajuda: a) "ligação telefônica"; b) "não possui".

- Restrição: Qual o tipo de restrição imposta: a) "acesso"; b) "ferramenta"; c) "equipamento"; d) "não possui".

- Monitoração: Qual a frequência de monitoramento das entidades quanto a sua localização ou situação: a) "tempo real", caso seja continuo; b) "parcial", caso seja momentâneo.

- Mitigação: Identifica o suporte aos métodos de busca por alternativas de mitigação, na ocorrência do precursor ou do acidente: a) "possui"; b) "não possui".

Como resultado, a Tab. 1, expressa o conteúdo relativo aos critérios em cada trabalho. Nesta tabela comparativa, nota-se a oportunidade para o desenvolvimento de um modelo adequado a diversos ambientes de trabalho, atenda os trabalhadores e também usuários; que notifique sobre os riscos de acidentes de forma compreensível, para a maioria dos usuários e que o modo de prevenção seja aplicado individual ou coletivamente, possuindo suporte ao atendimento de usuários que precisam de ajuda e ofereça recurso para mitigação.

Neste sentido, o modelo Ebenezer suporta diversos ambientes de trabalho, restringindo-se a questão da disponibilidade de Internet, atende aos usuários de um modo geral, usa o dispositivo tecnológico comum a maioria das pessoas, as notificações podem ser percebidas pelo modo vibratório, o modo de prevenção é aplicado individualmente ou coletivamente e realiza o monitoramento contínuo. As ressalvas são referentes à ausência da comunicação, para o atendimento aos usuários que precisam de ajuda e de métodos de busca por recursos para mitigação.

\section{Modelo Ebenezer}

Esta seção apresenta o Ebenezer $^{1}$, um modelo de prevenção de acidentes de trabalho, que sinaliza por meio de artefatos, os perigos decorrentes das atividades. Na sua concepção, são envolvidos princípios utilizados nas áreas da computação ubíqua, saúde ocupacional e do SWE.

\subsection{Visão geral do Ebenezer}

O Ebenezer emite notificações de alerta no smartphone das partes interessadas, a respeito dos riscos de acidentes presentes no ambiente ocupacional. Estes riscos provenientes das atividades ocupacionais executadas pelos trabalhadores, são identificados pelo Ebenezer, considerando as ferramentas utilizadas e a atividade ocupacional corrente.

A arquitetura do Ebenezer está ilustrada na Fig. 1, contendo os módulos de Acesso, Execução de atividades, Gerenciamento de atividades e de Alerta de perigos. Estes módulos, utilizam o serviço do banco de dados na nuvem FireBase ${ }^{2}$, para $o$ armazenamento ou a leitura dos dados, além disso os módulos, Gerenciamento de atividades e de Alerta de perigos utilizam a API Android Beacon Library ${ }^{3}$, que é uma biblioteca do Android para detectar beacons, configurada para a comunicação através do protocolo Altbeacon ${ }^{4}$

A descrição dos módulos que compõem o Ebenezer são os seguintes:

- Módulo de Acesso: Destinado ao funcionário, usuário e supervisor, tendo a responsabilidade de possibilitar o acesso destes na aplicação, através da inserção do nome e senha e do tipo de acesso;

- Módulo de Gerenciamento de atividades: Destinado ao Supervisor, tendo a responsabilidade de permitir o gerenciamento das atividades contendo funcionários, ferramentas e tipos

\footnotetext{
${ }^{1}$ Termo hebraico que significa pedra de ajuda ou gratidão. Fonte: https://www.significados.com.br/ebenezer/

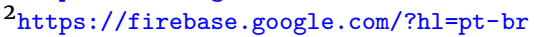

3 https://altbeacon.github.io/android-beacon-library/

4http://altbeacon.org/
} 
Tabela 1: Comparativo dos trabalhos

\begin{tabular}{l|l|l|l|l|l|l|l|l}
\hline $\begin{array}{l}\text { Critério / } \\
\text { Sistema }\end{array}$ & $\begin{array}{l}\text { Abran- } \\
\text { gência }\end{array}$ & $\begin{array}{l}\text { Dispo- } \\
\text { sitivo }\end{array}$ & $\begin{array}{l}\text { Notifi- } \\
\text { cação }\end{array}$ & Prevenção & Suporte & Restrição & $\begin{array}{l}\text { Moni- } \\
\text { toração }\end{array}$ & $\begin{array}{l}\text { Miti- } \\
\text { gação }\end{array}$ \\
\hline $\begin{array}{l}\text { Collision } \\
\text { accident (2010) }\end{array}$ & civil & próprio & textual & ambos & $\begin{array}{l}\text { não } \\
\text { possui }\end{array}$ & Não possui & $\begin{array}{l}\text { tempo } \\
\text { real }\end{array}$ & $\begin{array}{l}\text { não } \\
\text { possui }\end{array}$ \\
\hline $\begin{array}{l}\text { Proactive pre- } \\
\text { vention (2012) }\end{array}$ & civil & $\begin{array}{l}\text { próprio/ } \\
\text { servidor }\end{array}$ & $\begin{array}{l}\text { textual/ } \\
\text { luminoso }\end{array}$ & individual & $\begin{array}{l}\text { não } \\
\text { possui }\end{array}$ & $\begin{array}{l}\text { ferramenta/ } \\
\text { equipamento }\end{array}$ & $\begin{array}{l}\text { tempo } \\
\text { real }\end{array}$ & $\begin{array}{l}\text { não } \\
\text { possui }\end{array}$ \\
\hline $\begin{array}{l}\text { Risk manage- } \\
\text { ment (2012) }\end{array}$ & $\begin{array}{l}\text { civil/ } \\
\text { indústria }\end{array}$ & dashbord & tela & ambos & $\begin{array}{l}\text { não } \\
\text { possui }\end{array}$ & não possui & $\begin{array}{l}\text { tempo } \\
\text { real }\end{array}$ & $\begin{array}{l}\text { não } \\
\text { possui }\end{array}$ \\
\hline $\begin{array}{l}\text { Passage cons- } \\
\text { truction (2013) }\end{array}$ & $\begin{array}{l}\text { subter- } \\
\text { râneo }\end{array}$ & próprio & $\begin{array}{l}\text { textual/ } \\
\text { luminoso }\end{array}$ & ambos & $\begin{array}{l}\text { ligaça } \\
\text { telefônica }\end{array}$ & não possui & $\begin{array}{l}\text { tempo } \\
\text { real }\end{array}$ & possui \\
\hline $\begin{array}{l}\text { Gates } \\
\text { portals (2013) }\end{array}$ & específico & próprio & luminoso & individual & $\begin{array}{l}\text { não } \\
\text { possui }\end{array}$ & acesso & $\begin{array}{l}\text { tempo } \\
\text { real }\end{array}$ & $\begin{array}{l}\text { não } \\
\text { possui }\end{array}$ \\
\hline $\begin{array}{l}\text { Workplace } \\
\text { safety (2015) }\end{array}$ & específico & $\begin{array}{l}\text { smart- } \\
\text { watch }\end{array}$ & $\begin{array}{l}\text { textual/ } \\
\text { sonoro }\end{array}$ & individual & $\begin{array}{l}\text { não } \\
\text { possui }\end{array}$ & não possui & $\begin{array}{l}\text { tempo } \\
\text { real }\end{array}$ & $\begin{array}{l}\text { não } \\
\text { possui }\end{array}$ \\
\hline $\begin{array}{l}\text { Adaptive se- } \\
\text { curity (2015) }\end{array}$ & diversos & dashbord & tela & ambos & $\begin{array}{l}\text { não } \\
\text { possui }\end{array}$ & $\begin{array}{l}\text { acesso/ } \\
\text { ferramenta }\end{array}$ & $\begin{array}{l}\text { tempo } \\
\text { real }\end{array}$ & possui \\
\hline $\begin{array}{l}\text { Early War- } \\
\text { ning (2016) }\end{array}$ & civil & $\begin{array}{l}\text { smart- } \\
\text { phone }\end{array}$ & luminoso & individual & $\begin{array}{l}\text { não } \\
\text { possui }\end{array}$ & acesso & parcial & $\begin{array}{l}\text { não } \\
\text { possui }\end{array}$ \\
\hline $\begin{array}{l}\text { RAMIRES } \\
\text { (2016) }\end{array}$ & indústria & dashbord & tela & ambos & $\begin{array}{l}\text { não } \\
\text { possui }\end{array}$ & não possui & $\begin{array}{l}\text { tempo } \\
\text { real }\end{array}$ & possui \\
\hline
\end{tabular}

Fonte: Elaborado pelo autor

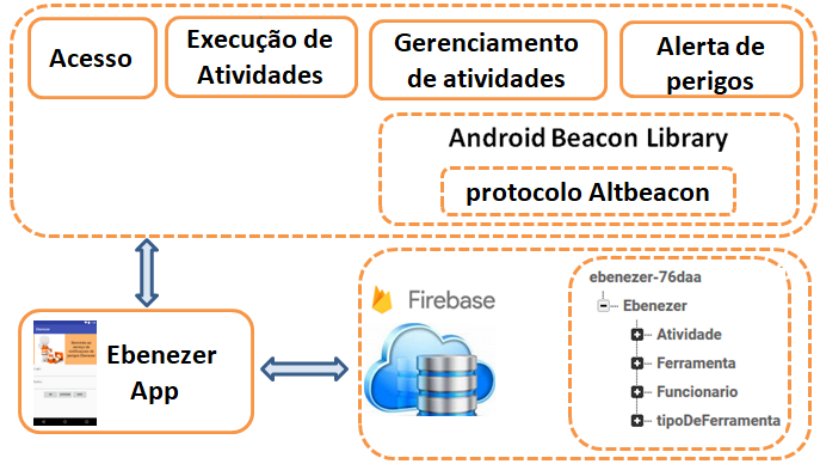

Figura 1: Arquitetura do modelo Ebenezer

de ferramentas, através da listagem, inclusão (seguindo o critério específico de cada um destes), ou remoção de algum dos objetos;

- Módulo de Execução de Atividades: Destinado ao usuário do tipo funcionário, sendo responsável por listar todas as atividades relacionadas a este funcionário, separar as que foram ativadas por ele, mostrar detalhes de qual(is) ferramenta(s) utilizar, e o(s) tipo(s) de perigo(s) que esta oferece;

- Módulo Alerta de perigos: Destinado ao usuário, tendo a responsabilidade de alertá-lo por meio de notificações e pelo modo vibratório contido no smartphone, a respeito dos perigos presentes no ambiente. O alerta é gerado pela comparação do UUID do beacon lido, com as identificações das ferramentas que estão sendo utilizadas pelos funcionários na execução das atividades.

\subsection{Diagrama de classes}

O diagrama de classes do modelo é baseada na NR1 (Ministério do Trabalho e Emprego; 2009) artigo 1.7(b,c), que determina ao empregador a elaboração das ordens de serviço para os empregados, constando neste documento, os riscos profissionais que possam originar-se nos locais de trabalho, enfatizando os

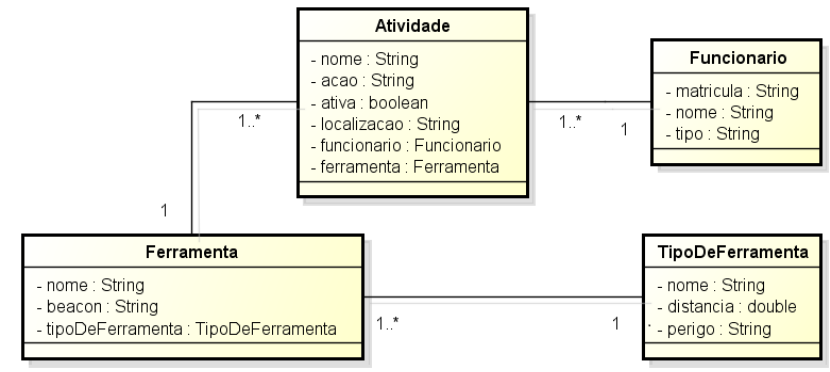

Figura 2: Diagrama de classes do Ebenezer

meios para prevenir e limitar tais riscos e as medidas adotadas.

A Fig. 2 mostra a estrutura contemplando as classes com suas relações e o tipo de atributo. Como visto, a classe Atividade tem um nome, uma ação que a representa, a situação da atividade, a localização, um funcionário e uma ferramenta. A classe Ferramenta tem um nome, a identificação do beacon a qual está associada e um TipoDeFerramenta. A classe TipoDeFerramenta tem um nome, uma distância segura e um perigo associado. A classe Funcionário tem nome, matrícula e tipo, podendo ser supervisor, funcionário ou usuário.

\section{Aspectos de implementação e avaliação}

Esta seção descreve os detalhes referentes à implementação do modelo Ebenezer, quanto aos artefatos utilizados no protótipo, sua interação com os usuários e a avaliação junto aos participantes envolvidos no processo, realizando os testes nos cenários descritos.

\subsection{Implementação}

O desenvolvimento do protótipo para smartphone é baseado nos casos de uso extraídos das normas regulamentadoras NR1 (Ministério do Trabalho e 


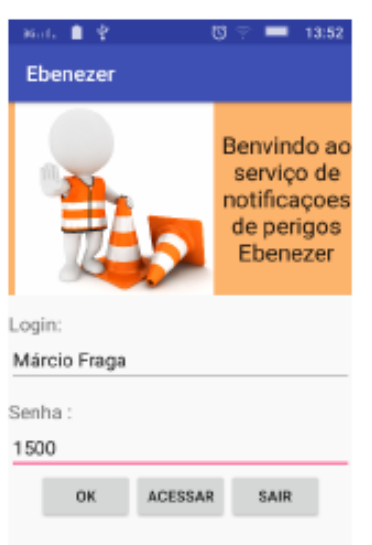

(a) Autenticação

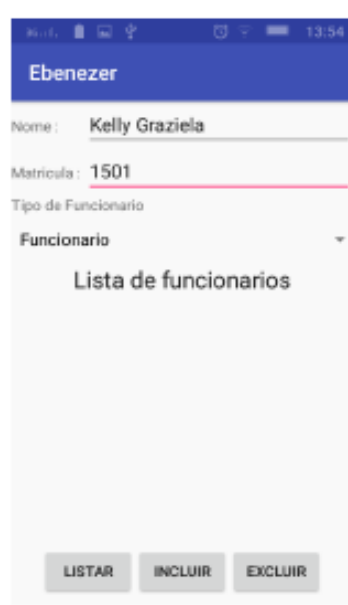

(b) Cadastro

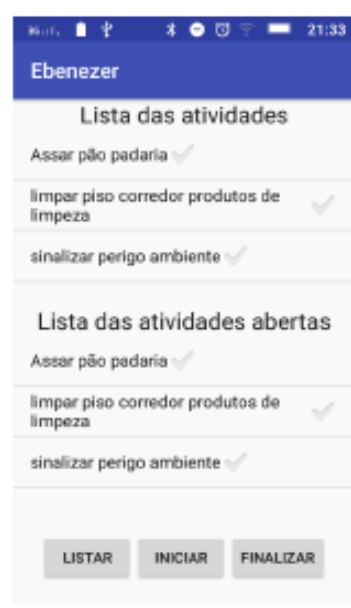

(c) Execução

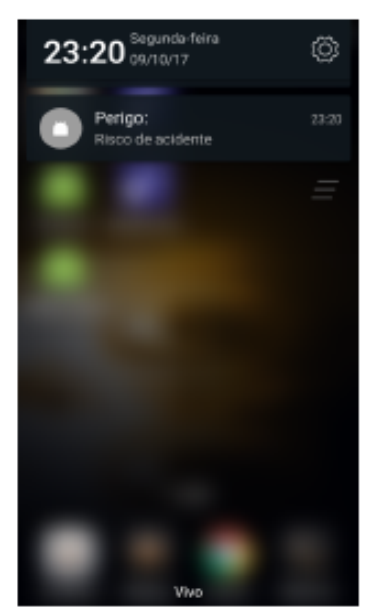

(d) Notificações

Figura 3: Telas de serviço do Ebenezer

Emprego; 2009), NR5 (Ministério do Trabalho e Emprego; 1999) e NR9 (Ministério do Trabalho e Emprego; 1994), onde foram levantados os requisitos necessários para a implementação da prevenção de acidentes.

O protótipo foi implementado, no ambiente de programação para dispositivos móveis Android Studio 2.3.3, utilizando a linguagem Java 8 e XML, compilado para o SDK API 23, android 6.0 (Marshmallow), e as bibliotecas appcompat-v7:25.3.0 e constraint-layout:1.0.1. Para a persistência e leitura dos dados é utilizado o Firebase incluindo as dependências firebase-database:10.2.6 e firebasecore:10.2.64 ao projeto. A API do Firebase dispõe da classe DatabaseReference para gravação direta dos dados e para a leitura é utilizado o método addValueEventListener que disponibiliza o dado atualizado em tempo real. Para a comunicação com o beacon é utilizado a API androidbeacon-library2.9.25, através da qual foi implementada a coleta do Identificador Único Universal (UUID), para realizar o registro do beacon associado à ferramenta, ou a sua comparação com a identificação dos beacons presentes nas ferramentas das atividades, para fins de alerta de perigos. Utiliza-se também um Beacon Ble Bluetooth Rfid Ativa de $28 \mathrm{~mm}$, com envio de pacotes a cada segundo e alcance de 8 metros em ambientes fechados e 25 metros em ambientes abertos ${ }^{5}$.

A Fig. 3 mostra as telas do protótipo nos testes reais, realizados nos cenários, onde os três atores (usuário, funcionário ou supervisor) realizam as interações no Ebenezer.

A autenticação (Fig. 3(a)) é realizada através da inserção do login e senha, caso este não esteja cadastrado, será direcionado a Fig. 3(b), onde realiza a inserção dos dados anteriores, acrescido da seleção do tipo, podendo ser um dos três atores. Sendo este um usuário, poderá fechar a tela de login (Fig. 3(a)) e receber as notificações em segundo plano (Fig. 3(d)), ou também fechar esta execução, clicando no botão SAIR, ou se for um funcionário, poderá listar, iniciar ou finalizar as atividades (Fig. 3(c)).

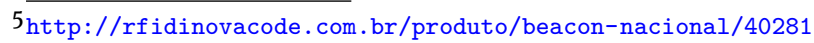

Por fim, se for um supervisor, este poderá optar (Fig. 4(a)) por gerenciar funcionários, realizando os mesmos passos da tela de cadastro (Fig. 3(b)), ou gerenciar os tipos de ferramentas (Fig. 4(b)), através da inclusão inserindo um nome, uma distância segura, e um respectivo perigo.

Ainda pode incluir a ferramenta inserindo um nome, selecionando o tipo de ferramenta e associando a identificação do beacon à ferramenta, através do botão IDENTIFICAR FERRAMENTA, estando o smartphone próximo ao beacon. Além disso, têm a possibilidade de listar as ferramentas para verificação do número de identificação e do perigo associado a ela e fazer as exclusões. Também pode gerenciar as atividades (Fig. 4(d)), inserindo um nome, uma ação que a represente, o objeto de intervenção, o local, selecionando o funcionário e a ferramenta, podendo ainda listá-las para a verificação do perigo associado, ou fazer as exclusões.

\subsection{Avaliação}

Seguindo à abordagem da comunidade acadêmica para a validação de sistemas Ubíquos (Satyanarayanan; 2001) e (Tavares et al.; 2015), o modelo foi avaliado em dois cenários: o cenário $\mathrm{A}$ descreve um risco de acidente proveniente de uma atividade cuja localização pode alterar ao longo tempo. Por sua vez, o cenário $\mathrm{B}$ aborda um risco de acidente proveniente de uma atividade que utiliza o mesmo espaço ao longo do tempo.

\subsubsection{Cenário $A$}

"Márcio Fraga (Fig. 5(a)) é proprietário de um supermercado localizado no bairro Timbaúva, na cidade de Montenegro RS, na qual ele tem cinco colaboradores no seu quadro de funcionários, que desempenham variadas atividades (atendimento no caixa, padaria, açougue, limpeza do estabelecimento, etc). Preocupado em prevenir acidentes com os funcionários, clientes e fornecedores, para manter uma boa reputação do seu estabelecimento, Márcio opta em utilizar o aplicativo Ebenezer para esta finalidade. Então ele se autentica no Ebenezer com a sua credencial (Fig. 3(a)), como ainda não 


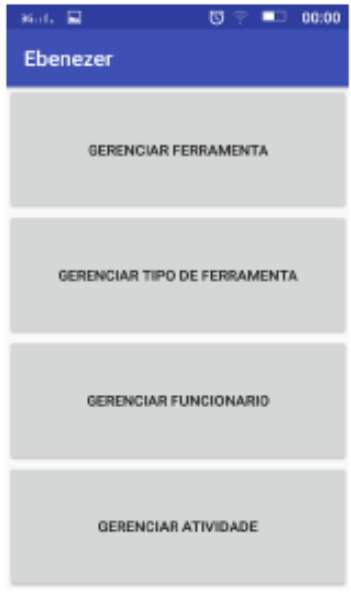

(a) Seleção

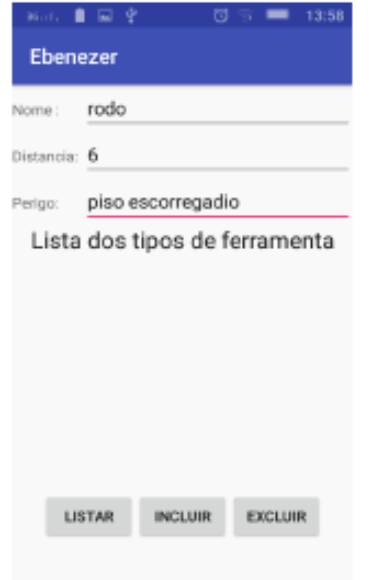

(b) Tipo de ferramenta

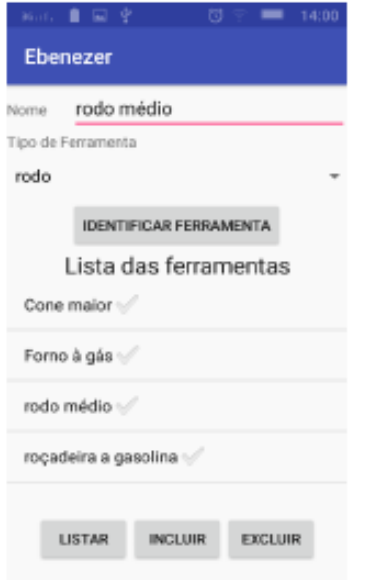

(c) Ferramenta

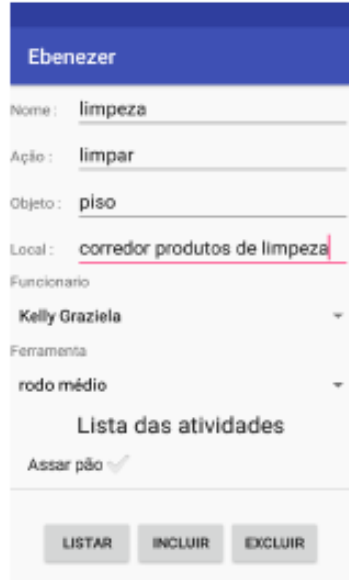

(d) Atividade

Figura 4: Telas de gerenciamento do Ebenezer

tem cadastro, o Ebenezer, libera a tela de cadastro (Fig. 3(b)), para ele inserir suas credencias e o tipo de acesso como supervisor, então ele volta para tela de autenticação (Fig. 3(a)) e realiza o acesso.

Agora ele pode gerenciar os funcionários, tipos de ferramenta, ferramentas e atividades (Fig. 4(a)), começando pelo funcionário (Fig. 3(b)), ele registra a funcionária Kelly (Fig. 5(b)), depois os tipos de ferramentas rodo e cone (Fig. 4(b)), com decorrentes perigos "Piso escorregadio" e "Risco de acidente", as ferramentas (Fig. 4(c)) rodo médio e cone maior (Fig. 5(c,d)), e as atividades (Fig. 4(d)) "limpar piso corredor produtos de limpeza" e "sinalizar perigo no ambiente" (Fig. 5(b,d)).

Desta forma, a funcionária Kelly pode e realiza a autenticação com sua credencial no Ebenezer (Fig. 3(a)), lista a(s) atividade(s) designada(s) a ela (Fig. 3(c)), que seleciona a atividade "limpar piso corredor produtos de limpeza", na qual mostra a ferramenta necessária e o decorrente perigo. Kelly pega as ferramentas descritas para a atividade, chega ao local e inicia a tarefa (Fig. 3(c), 5(b)), depois de um certo tempo, Kelly finaliza esta atividade (Fig. 3(c)), porém ela identifica que o piso ainda oferece certo risco de acidente por estar molhado, então ela coloca o cone de sinalização (Fig. 5(d)) e inicia a atividade "sinalizar perigo no ambiente" (Fig. 3(c)).

Posteriormente a cliente Denise que é um usuário e está autenticada no serviço Ebenezer (Fig. 3(a)), acessa as dependências deste supermercado (Fig. 5(e)), chegando próximo à área onde está ocorrendo a atividade realizada pela funcionária Kelly, o seu smartphone vibra e então ela visualiza a mensagem "Perigo: Risco de acidente." (Fig. 3(d)), ficando assim, ciente da situação, ela toma os devidos cuidados para acessar este corredor onde está a mercadoria que veio comprar (Fig. 5(f)). Mais tarde, Kelly verifica o piso e identifica que não há mais riscos de acidentes, então finaliza a execução da atividade (Fig. 3(c)) e recolhe o cone que foi utilizado nesta tarefa (Fig. 5(g))."

\subsubsection{Cenário $B$}

"O mesmo proprietário Márcio (Fig. 6(a)) tem em seu estabelecimento o setor da padaria, neste

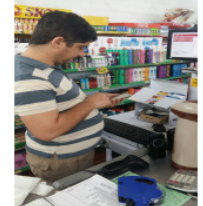

(a) Supervisor

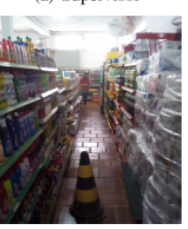

(e) Usuário

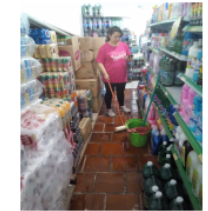

(b) Funcionário

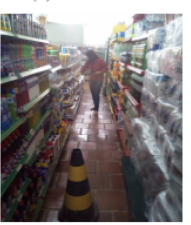

(f) Usuário

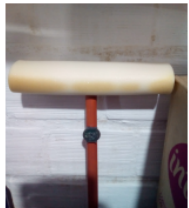

(c) Ferramenta

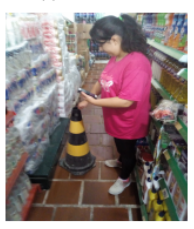

(g) Funcionário

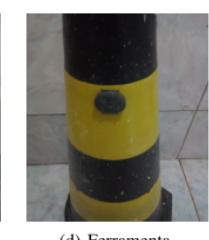

(d) Ferramenta

Figura 5: Interação dos atores no cenário A

local ocorrem situações de riscos de acidentes. Para prevenir estas ocorrências, Márcio registra o forno como um tipo de ferramenta (Fig. 4(b)) com decorrente perigo "risco de queimadura", a ferramenta forno a gás (Fig. 4(c), 6(b)) e então cria a atividade (Fig. 4(d)) "Assar pães setor da padaria", designando a funcionária Kelly (Fig. 6(c)) e selecionando a ferramenta forno a gás para este fim. A funcionária Kelly, estando já autenticada (Fig. 3(a)), lista novamente as atividades (Fig. 3(c)), onde aparece a nova atividade designada a ela, que é "Assar pães setor da padaria", Kelly então a seleciona e inicia essa atividade (Fig. 6(c)).

Posteriormente o funcionário Gabriel (Fig. 6(d)), que também está autenticado no serviço Ebenezer como usuário (Fig. 3(a)), carrega mercadorias para abastecer o depósito, cujo acesso é próximo ao forno na qual são assados os pães. Estando ele nas proximidades deste forno (Fig. 6(d)), o seu smartphone vibra, então ele para, e visualiza a mensagem "Perigo: risco de queimadura" (Fig. 3(d)). Ciente da situação, toma os devidos cuidados para acessar em segurança o depósito para descarregar as mercadorias. Após decorrido o tempo desta atividade Kelly verifica que o forno não oferece mais risco de queimadura e a finaliza (Fig. 3(c), 6(e))." 
Tabela 2: Questionário de avaliação do modelo

\begin{tabular}{c|l}
\hline Questão & Afirmação \\
\hline 1 & O aplicativo Ebenezer é de fácil compreensão. \\
\hline 2 & Não haveria dificuldade em utilizar o aplicativo Ebenezer. \\
\hline 3 & Não é necessário esforço significativo para utilizar as funcionalidades oferecidas pelo Ebenezer. \\
\hline 4 & Não é necessária experiência significativa no uso dos recursos tecnológicos oferecidos pelo Ebenezer. \\
\hline 5 & As informações sobre os perigos são apresentadas de forma clara e objetiva, permitindo a compreensão. \\
\hline 7 & A notificação de perigo do Ebenezer me ajudaria a ter mais atenção com os riscos de acidentes. \\
\hline 8 & O uso da parte de gerenciamentos do Ebenezer auxiliaria na organização das atividades. \\
\hline 9 & O uso da parte da execução de atividades pelo funcionário o auxiliaria no planejamento da execução. \\
\hline 10 & O Ebenezer seria útil para auxiliar as pessoas a se prevenirem dos riscos de acidentes. \\
\hline
\end{tabular}

Fonte: Elaborado pelo autor

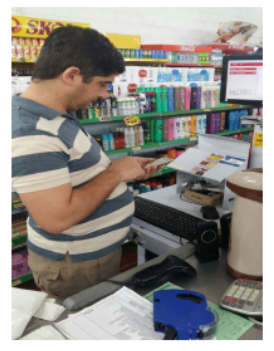

(a) Supervisor

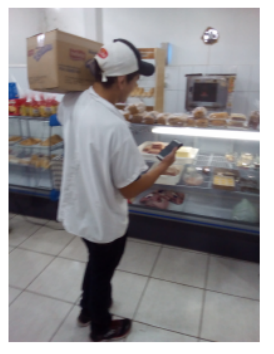

(d) Usuário

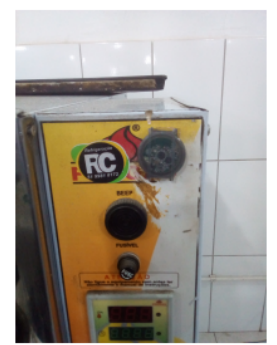

(b) Ferramenta

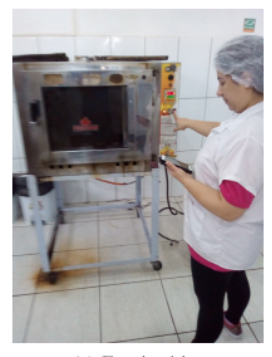

(e) Funcionário

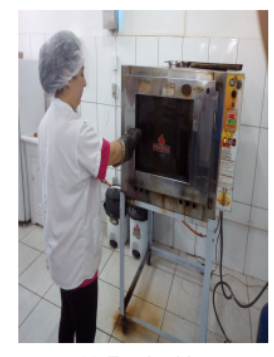

(c) Funcionário
Figura 6: Interação dos atores no cenário B

\section{Resultados}

A validação do modelo consistiu no desenvolvimento e na aplicação de um questionário realizado na ferramenta Google formulários, contendo então, as perguntas relativas ao perfil do entrevistado, e aos parâmetros de facilidade de uso e utilidade percebida pelo usuário, adotados no Modelo de Aceitação de Tecnologia (Stephanidis and Savidis; 2001).

Neste questionário os entrevistados responderam questões destinadas para análise do seu perfil, retratando a respeito da sua faixa etária, sexo, escolaridade, grau de experiência com recursos tecnológicos, ramo de atividade e área de atuação, e também para análise de aceitação de tecnologia. Para cada afirmação referente aos parâmetros de aceitação de tecnologia, o entrevistado poderia assinalar com "Concordo plenamente", "Concordo", "Indiferente", "Discordo" ou "Discordo plenamente" e por último, poderia deixar os comentários livres a respeito do modelo. Como visto na Tab. 2, as cinco primeiras questões são referentes ao parâmetro de facilidade de uso percebida e as demais abrangendo ao parâmetro de utilidade percebida.

A aplicação deste questionário foi feito em duas fases distintas: na primeira, o questionário foi disponibilizado via rede de mensagens whatsapp, ao público envolvido nos dois cenários. Na segunda etapa, foi apresentado o protótipo aos profissionais da área de gestão (gerentes, supervisores), com formação técnica em mecânica e de engenharia mecânica, elétrica e produção. Também a profissionais na área de operação (manutenção, produção, planejamento) com nível técnico e ensino médio, e especialistas na área da segurança (técnicos e engenheiro).

A demonstração envolveu toda a parte de cadastro, a parte de execução relativa ao funcionário e a parte de recebimento das notificações de perigo, sendo a execução simulada em um cenário peculiar a cada um deles, posteriormente foi disponibilizado a estes, o questionário através de emails.

O questionário, relativo à primeira fase da pesquisa, contabilizou quatro participantes, conforme visualizado na Fig. 7, o perfil deles é o seguinte: a faixa etária é entre 15 e 45 anos (Fig. 7(a)), não havendo disparidade entre sexos $50 \%$ (Fig. 7(b)), na questão escolaridade (Fig. 7(c)), 25\% com ensino fundamental, $50 \%$ com ensino médio e $25 \%$ com ensino superior incompleto. Trabalham no ramo do comércio $75 \%$ e prestação de serviços $25 \%$ (Fig. 7(d)), 25\% atuam na área de gerenciamento (Fig. 7(e)), $50 \%$ na de operação e $25 \%$ em outra, $75 \%$ com médio grau de experiência com recursos tecnológicos contra $25 \%$ de regular (Fig. $7(\mathrm{f})$ ).

Os participantes como visto na Fig. 8, foram unânimes, no quesito facilidade de uso percebida (Fig. 8(a)), 55\% concordaram plenamente e 45\% concordaram. No quesito utilidade percebida (Fig. 8(b)), 60\% concordaram plenamente e $40 \%$ concordaram. Os resultados positivos também foram mostrados nos comentários livres a respeito da sua utilidade. "Melhoraria o controle das atividades para os funcionários", "Auxiliaria nos lembretes para não haver esquecimento de alguma ferramenta", "Me ajudaria a ter mais cuidado nos momentos de desatenção".

$O$ resultado da segunda fase da pesquisa como visto na Fig. 9, contabilizou 14 participantes com o seguinte perfil: $100 \%$ trabalham na indústria, todos do sexo masculino, situação condizente segundo o levantamento (Economia e Emprego; 2017), a qual mostra que a maioria dos homens trabalham na indústria de transformação.

Os participantes tinham uma faixa etária (Fig. 9(a)) entre 26 e 70 anos, com maior índice nas faixas de 36 a 40 anos com $29 \%$ e 46 a 50 anos também com $29 \%$, seguida da faixa de 26 a 30 anos com 


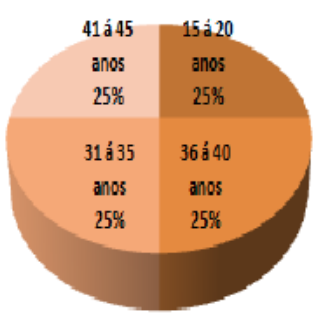

(a) Faixa etária

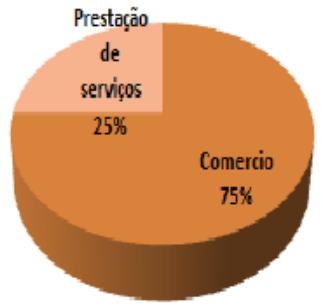

(d) Ramo de atividade

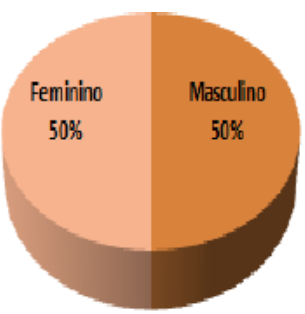

(b) Sexo

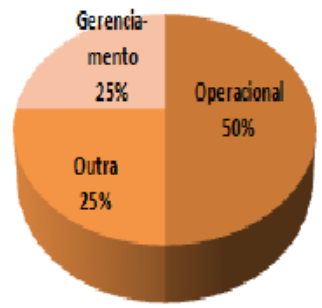

(e) Área de atuação

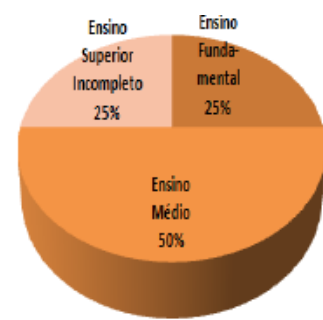

(c) Escolaridade

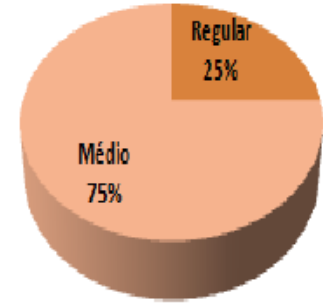

(f) Conhecimento / Experiência em informática

Figura 7: Perfil dos entrevistados da primeira fase

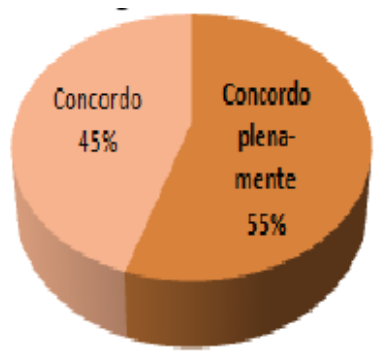

(a) Facilidade de uso percebida

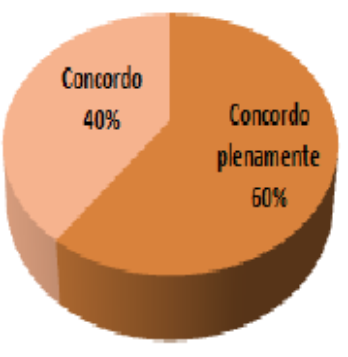

(b) Utilidade percebida

Figura 8: Resultado de aceitação da primeira fase

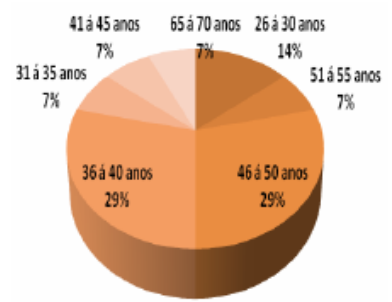

(a) Faixa etária

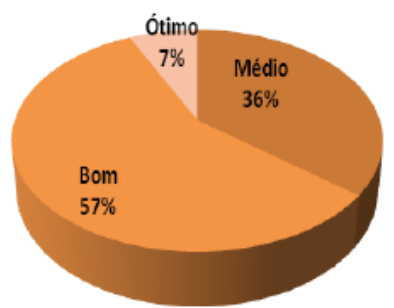

(c) Conhecimento ou Experiência em informática

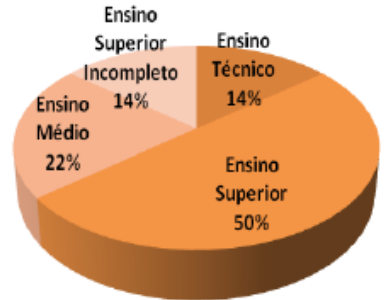

(b) Escolaridade

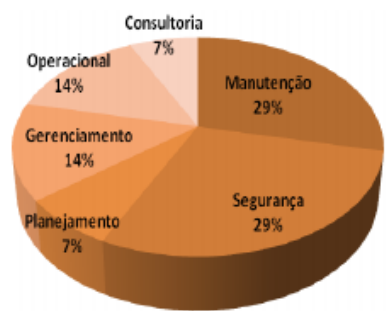

(d) Área de atuação

Figura 9: Perfil dos entrevistados da segunda fase

$14 \%$, e as restantes faixas etárias com $7 \%$. Na questão escolaridade (Fig. 9 (b)), 50\% têm o ensino superior, $22 \%$ o ensino médio, $14 \%$ o ensino superior incompleto e $14 \%$ o ensino técnico. Atuam nas área de

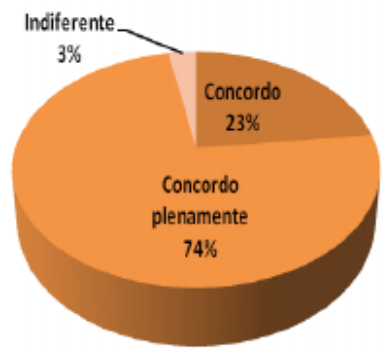

(a) Facilidade de uso percebida

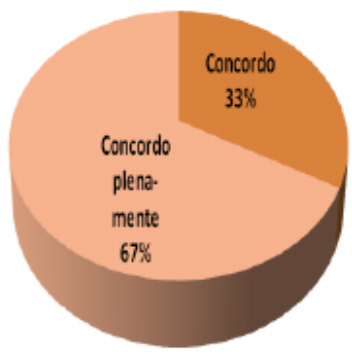

(b) Utilidade percebida
Figura 10: Resultado da aceitação da segunda fase

segurança 29\% (Fig. 9(d)), manutenção também com $29 \%$, gerenciamento $14 \%$, operação $14 \%$, consultoria $7 \%$ e planejamento de manutenção também com $7 \%$. Com relação ao conhecimento ou experiência com recursos tecnológicos (Fig. 9(c)), cerca de 7\% têm um ótimo grau, seguidos de $57 \%$ de bom e $36 \%$ de médio grau.

0 resultado de aceitação mostrado na Fig. 10, aplicado aos participantes da segunda fase de avaliação do modelo, mostrou alto nível de aceitação, no quesito facilidade de uso percebida (Fig. 10(a)), $74 \%$ concordaram plenamente, $23 \%$ concordaram e $3 \%$ indiferentes. No quesito utilidade percebida (Fig. 10(b)), 67\% concordaram plenamente e 33\% concordaram, resultado também evidenciado nos comentários.

Nos comentários livres destes entrevistados, houve uma maior participação, por se tratar de um público com conhecimentos ligados diretamente ou indiretamente a área de segurança do trabalho, onde foram sugeridas melhorias, observado oportunidades e utilidades.

Na parte de sugestões de melhorias, comentou-se a questão de possibilitar a inserção de perigos pelos executantes das atividades, para obtenção de um mapeamento mais apurado dos perigos e comportar o histórico dos riscos e integração com o setor de segurança. Outra sugestão que poderia ser agregado 
é a permissão de trabalho à atividade, onde constam as partes envolvidas e as recomendações de segurança ao funcionário.

Os entrevistados citaram que seria interessante agregar ao protótipo a lista dos Epis necessários para a execução da atividade e só liberá-la para a execução mediante as documentações estarem regularizadas (atestados de saúde, treinamentos específicos) e descrever os perigos para o executante da atividade. Ressaltaram que o aplicativo deveria assegurar as questões de segurança no acesso a áreas classificadas, que requerem que os aparelhos sejam intrinsecamente seguros.

$\mathrm{Na}$ questão de utilidade, comentou-se que o protótipo atende alguns itens referentes a NR1 sobre as ordens de serviço e a NR9 referente ao Programa de Prevenção de Riscos Ambientais, citando-se os exemplos de utilização em uma máquina de processamento florestal, que tem um raio de atuação de 70 metros. Outra utilização seria nas inspeções de solda por raios $X$, que da mesma forma tem raio de atuação de longo alcance que requerem, segundo a NR12, sinalização para alertar as pessoas a respeito do risco de acidente de trecho em trecho, tornando o processo bastante dispendioso. Salientou-se que seria mais um acessório para agregar nas práticas de prevenção de acidentes.

Nas oportunidades, observou-se que o aplicativo pode se tornar uma ferramenta de auxilio relativo à segurança operacional nas empresas, inclusive relativo a NR12 do Ministério do Trabalho, podendo monitorar o tempo e a performance que o empregado levou para executar uma determinada atividade evitando acidentes, desperdício de tempo e perdas no processo produtivo.

\section{Conclusão}

Esse trabalho apresentou o modelo Ebenezer para prevenção de acidentes, interagindo em cenários de atividades reais exercidas pelos trabalhadores.

O Ebenezer caracteriza-se por identificar e registrar as atividades, notificar as partes interessadas sobre os perigos presentes no ambiente de trabalho, a fim de prevenir os riscos de acidentes. Baseado nos estudos dos trabalhos relacionados ao tema, notou-se a oportunidade de desenvolvimento de um modelo para prevenção de acidentes, tendo o potencial para atuar em várias áreas, sendo um aplicativo instalado em dispositivos móveis (smartphones), possibilitando de modo simplificado, dinâmico e espontâneo, a interação dos trabalhadores e usuários, com este recurso tecnológico.

Para comprovar a utilidade do modelo foram feitos testes reais com as partes envolvidas (supervisor, funcionário, usuário) e principalmente a validação junto a especialistas que atuam na área de segurança e gestão e operação com conhecimento específico na prevenção de acidentes. Com base nos resultados da pesquisa de aceitação do modelo com estas pessoas, pode concluir-se que houve a aceitação.

Os resultados mostram que a solução possui utilidade, possibilitando que a tecnologia desenvolvida possa ser aprimorada, para tornar-se um acessório de segurança para prevenção de acidentes com os usuários, como também auxiliar os supervisores na distribuição das atividades, os funcionários na utilização de EPIs e ferramentas e a integração da equipe de segurança na fiscalização das atividades.

A principal contribuição científica deste trabalho é a especificação de um modelo para a prevenção de acidentes de trabalho, relativo à identificação e divulgação dos perigos resultantes das atividades executadas pelo trabalhador, promovendo assim a prevenção de acidentes de trabalho. Outra contribuição é que o modelo pode ser adaptado para aplicações sensíveis ao contexto. Como contribuição socioeconômica, pode-se mencionar o desenvolvimento de mais um recurso para prevenção, consequentemente a redução no número de acidentes de trabalho.

Baseado nos estudos desse modelo, surgem novas possibilidades para a continuidade com trabalhos futuros, tais como: o aprimoramento do modelo na inclusão das restrições; quanto ao uso de ferramentas, EPIs e execução de atividades, levando-se em conta as aptidões de saúde e treinamentos do funcionário; inclusão dos registros de perigos de incidentes ou acidentes relatados pelos funcionários e usuários, para realizar inferências quanto a sugestão de recursos às partes interessadas a fim de atuar na mitigação; comparar o modelo aos métodos convencionais de prevenção de acidentes.

\section{Agradecimentos}

Os autores agradecem à Fundação de Amparo à Pesquisa do Estado do Rio Grande do Sul (FAPERGS), ao Conselho Nacional de Desenvolvimento Científico e Tecnológico (CNPq) e à Universidade do Vale do Rio dos Sinos (Unisinos) pelo apoio ao desenvolvimento desse trabalho.

\section{Referências}

Almeida, I. M. d. and Jackson Filho, J. M. (2007). Acidentes e sua prevenção, Revista Brasileira de Medicina do Trabalho 32(115): 7-18. Disponível em http://www.scielo.br/pdf/rbso/v32n115/02.pdf (Acessado em 22 Novembro 2017).

Bastos-Ramos, T. P., Santana, V. S. and Ferrite, S. (2015). Estratégia saúde da família e notificações de acidentes de trabalho, brasil, 2007-2011, Epidemiologia e Serviços de Saúde 24(4): 641650. http://www.scielo.br/pdf/ress/v24n4/ 2237-9622-ress-24-04-00641.pdf.

Chae, S. and Yoshida, T. (2010). Application of RFID technology to prevention of collision accident with heavy equipment, Automation in Construction 19(3): 368-374. http://dx.doi.org/10 $1016 / j$. autcon .2009 .12 .008 .

Computing, A. et al. (2006). An architectural blueprint for autonomic computing, IBM White Paper 31: 1-6. Disponível em http: //citeseerx.ist.psu.edu/viewdoc/download?doi= 10.1.1.150.1011\&rep=rep1\&type=pdf (Acessado em 25 Novembro 2017).

Ding, L. Y., Zhou, C., Deng, Q. X., Luo, H. B., Ye, X. W., Ni, Y. Q. and Guo, P. (2013). Real-time 
safety early warning system for cross passage construction in Yangtze Riverbed Metro Tunnel based on the internet of things, 36: 25-37. http: //dx.doi.org/10.1016/j.autcon.2013.08.017.

Economia e Emprego (2017). Mulheres ganham espaço no mercado de trabalho, Governo do Brasil. Disponível em http: //www.brasil.gov.br/economia-e-emprego/2017/03/ mulheres-ganham-espaco-no-mercado-de-trabalho (Acessado em 3 Novembro 2017).

Fugini, M., Raibulet, C. and Ubezio, L. (2012). Risk assessment in work environments: modeling and simulation, Concurrency and computation: Practice and experience 24(18): 2381-2403. http://onlinelibrary.wiley.com/doi/10.1002/ cpe.2828/full.

Fugini, M., Teimourikia, M. and Hadjichristofi, G. (2016). A web-based cooperative tool for risk management with adaptive security, Future Generation Computer Systems 54: 409-422. http: //dx.doi.org/10.1016/j.future.2015.04.015.

Gerhardt, T. E. and Silveira, D. T. (2009). Métodos de pesquisa, Editora da UFRGS, Porto Alegre, RS, BR. Disponível em http://www.ufrgs.br/ cursopgdr/downloadsSerie/derad005.pdf (Acessado em 23 Novembro 2017).

Kelm, A., Laußat, L., Meins-becker, A., Platz, D., Khazaee, M. J., Costin, A. M., Helmus, M. and Teizer, J. (2013). Mobile passive Radio Frequency Identification ( RFID ) portal for automated and rapid control of Personal Protective Equipment ( PPE ) on construction sites, Automation in Construction 36: 38-52. http://dx.doi.org/10.1016/ j. autcon.2013.08.009.

Kritzler, M., Tenfält, A., Bäckman, M. and Michahelles, F. (2015). Wearable Technology as a Solution for Workplace Safety, Proceeding of the 14th International Conference on Mobile and Ubiquitous Multimedia (MUM 2015) pp. 213-217. https ://dl . acm. org/citation. $\mathrm{cfm}$ ?id=2836062.

Lin, X. Y., Ho, T. W., Fang, C. C., Yen, Z. S., Yang, B. J. and Lai, F. (2015). A mobile indoor positioning system based on ibeacon technology, 2015 37th Annual International Conference of the IEEE Engineering in Medicine and Biology Society (EMBC), pp. 4970-4973. https://ieeexplore.ieee.org/ document/7319507/.

Maia, A., Saito, C., Oliveira, J., Bussacos, M., Maeno, M., Lorenzi, R. et al. (2016). Acidentes de trabalho no brasil em 2013: comparação entre dados selecionados da pesquisa nacional de saúde do ibge (pns) e do anuário estatístico da previdência social (aeps) do ministério da previdência social., Fundacentro/Serviço de Estatística e EpidemiologiaSEE . Disponível em http://www.fundacentro. gov.br/arquivos/projetos/estatistica/boletins/ boletimfundacentro1vfinal.pdf (Acessado em 20 Novembro 2017).

Ministério do Trabalho e Emprego (1994). NR 9 PROGRAMA DE PREVENÇÃO DE RISCOS AMBIENTAIS, Governo do Brasil, Brasilia, Brasil. Disponível em
http://www.trabalho.gov.br/images/Documentos/ SST/NR/NR9.pdf (Acessado em 25 Novembro 2017).

Ministério do Trabalho e Emprego (1999). NR 5 COMISSÃO INTERNA DE PREVENÇÃO DE ACIDENTES, Governo do Brasil, Brasilia, Brasil. Disponível em http://trabalho.gov.br/images/Documentos/SST/NR/ NR5.pdf (Acessado em 26 Novembro 2017).

Ministério do Trabalho e Emprego (2009). NR 1 DISPOSIÇÕES GERAIS, Governo do Brasil, Brasilia, Brasil. Disponível em http://trabalho.gov.br/ images/Documentos/SST/NR/NR1.pdf (Acessado em 25 Novembro 2017).

Podgórski, D., Majchrzycka, K., Dabrowska, A., Gralewicz, G. and Okrasa, M. (2016). Towards a conceptual framework of osh risk management in smart working environments based on smart ppe, ambient intelligence and the internet of things technologies, International Journal of Occupational Safety and Ergonomics 23(1): 1-20. https://doi.org/ $10.1080 / 10803548.2016 .1214431$.

Satyanarayanan, M. (2001). Pervasive computing: Vision and challenges, IEEE Personal communications 8(4): 10-17. http://ieeexplore.ieee.org/abstract/ document/943998/.

Simonelli, A. P. and Camarotto, J. A. (2011). As políticas brasileiras e internacionais de incentivo ao trabalho de pessoas com deficiência: uma reflexão, Cadernos de Terapia Ocupacional da UFSCar 19(3): 333342. http://www. cadernosdeterapiaocupacional. ufscar.br/index.php/cadernos/article/view/503.

Stephanidis, C. and Savidis, A. (2001). Universal access in the information society: methods, tools, and interaction technologies, Universal access in the information society 1(1): 40-55. https://link. springer.com/article/10.1007\%2Fs102090100008.

Tavares, J., Barbosa, J., Cardoso, I., Costa, C., Yamin, A. and Real, R. (2015). Hefestos: an intelligent system applied to ubiquitous accessibility, Universal Access in the Information Society 15(4): 589-607. https : //link. springer.com/ article/10.1007/s10209-015-0423-2.

Teimourikia, M. and Fugini, M. (2016). Ontology development for run-time safety management methodology in Smart Work Environments using ambient knowledge, Future Generation Computer Systems 68: 428-441. http://dx.doi.org/10.1016/j future.2016.07.003.

Yang, H., Chew, D. A. S., Wu, W., Zhou, Z. and Li, Q. (2012). Design and implementation of an identification system in construction site safety for proactive accident prevention, Accident Analysis and Prevention 48: 193-203. http://dx. doi.org/10. 1016/j.aap. 2011.06.017.

Yi, W., Chan, A. P. C., Wang, X. and Wang, J. (2016). Development of an early-warning system for site work in hot and humid environments: A case study, Automation in Construction 62: 101-113. http://dx. doi.org/10.1016/j. autcon.2015.11.003. 